\title{
CTGF Positively Regulates BMP12 Induced Tenogenic Differentiation of Tendon Stem Cells and Signaling
}

\author{
Junpeng Liua Xu Tao ${ }^{a}$ Lei Chen ${ }^{b}$ Weifeng Han ${ }^{c}$ You Zhoua Kanglai Tanga \\ aDepartment of Orthopaedics, Southwest Hospital, Third Military Medical University, \\ Chongqing, 'D Department of Orthopaedics, Wuhan General Hospital of Guangzhou Military Region, \\ Wuhan, 'Department of Orthopaedics, Beijing Tian Tan Hospital, Capital medical University, Beijing, China
}

\section{Key Words}

Tendon stem cells (TSCs) • CTGF • BMP-12/ Smad1/5/8 pathway • Tenogenic differentiation

\begin{abstract}
Background/Aims: Disordered differentiation of tendon stem cells (TSCs) during repair of injured tendon can result in the pathogenesis of chronic tendinopathy. Understanding tenocyte differentiation may provide new therapeutic insights for the prevention and treatment of chronic tendinopathy. The aim of our study was to determine if CTGF exerts a similar effect on BMP12-driven differentiation of rat TSCs. Methods: In overexpressing and RNA interference CTGF TSCs, tenogenic differentitation and the expression of related genes were determined by immunofluorescence staining, quantitative $P C R$, and western blotting, with or without BMP12 treatment. The interaction in vitro between CTGF and BMP12 was detected by Chemical crosslinking assay. Results: Our results showed that BMP12 effectively increased the expression of the tenocyte lineage markers scleraxis (Scx) and tenomodulin (Tnmd) at both mRNA and protein levels. Over-expression of CTGF from a lentiviral vector increased the expression of Scx and Tnmd as well as tendon proteins type I collagen (ColI) and tenascin-C (Tn-C) in TSCs compared to non-treated control cells with or without simultaneous BMP12 stimulation. Knockdown of CTGF expression decreased the expression of Scx, Tnmd, ColI and $\mathrm{Tn}-\mathrm{C}$ compared to control cells. Chemical crosslinking experiments demonstrated a direct interaction between CTGF and BMP12. Conclusion: In conclusion, BMP12 plays a crucial role in tenogenesis via the Smad1/5/8 pathway, and CTGF positively promotes this effect.
\end{abstract}

J. Liu and X. Tao contributed equally. 


\section{Introduction}

Tendons are fibrous connective tissue bands which attach muscle to bone and convey mechanical force, permitting joint and whole body movement. As a result of constant mechanical loading, tendons are frequently subject to various pathologies, collectively called tendinopathies, typically involving inflammation and degradation of connective tissue. Common histopathological symptoms of tendinopathies include tissue calcification, mucoid degeneration and lipid cell accumulations $[1,2]$. Musculoskeletal disorders, of which tendinopathies constitute a substantial proportion, cost over $\$ 950$ billion in the U.S. alone in 2006. The economic costs for treatment of tendinopathy can be attributed in part to the poor clinical outcomes resulting from the inferiority of repaired compared to healthy tendons, and the resistance of injured tendon tissue to regeneration because of its hypocellular and hypovascular structure [3]. Therefore, cell-based therapies are an attractive future avenue for tendinopathy treatment.

Tendons consist of fascicles composed of collagen fiber bundles with tenocytes interposed between the fascicles [2]. Tendon fibroblasts play a key role as parenchymal cells [4] in repairing extracellular matrix but are fully differentiated cells difficult to prepare for cell-based therapy [3]. Tendon stem cells (TSCs) or progenitor cells have been identified in mouse, rat and human tendon tissue $[5,6]$ which can differentiate into fibroblasts, as well as other cell types [7]. Rabbit TSCs have been shown to differentiate into adipocytes, chondrocytes and osteocytes in vitro and to form tissues with characteristics of tendon, cartilage and bone in vivo [8]. However, the molecular signals which induce and regulate tenocyte differentiation are still poorly understood. Characterization of chick and mouse tendon differentiation in vivo reveals increased expression of connective tissue-specific basic helix-loop-helix transcription factor scleraxis (Scx) [9], beginning on embryonic day 9 in the mouse and continuing into adulthood[10-12]. Scx in turn induces expression of tenascin-c, a glycoprotein specifically present in developing tendon $[9,13]$. Isolated TSCs from rat tendon were reported to express elevated levels of mRNA for Scx and tenomodulin (Tnmd), another marker of tenocyte differentiation [14], as well as increased transcription of type I collagen (ColI) and associated proteoglycans decorin and biglycan [15].

A key molecule driving tenogenesis is Bone Morphogenic Protein 12 (BMP12), initially described by Storm et al. in mouse as growth/differentiation factor 7 [16]. Human BMP12, as well as BMP13 and 14, have been shown to induce formation of new connective tissue rich in type I collagen in a rat model utilizing ectopic implantation of BMP12-infused bone matrix particles [17]. BMP12 has been successfully employed to repair and increase tensile strength in a rat Achilles tendon rupture model [18] and to repair chicken flexor tendon laceration [19]. Like other members of the TGF- $\beta$ superfamily, BMP12 signals via heteromeric complexes of Bone Morphogenic Protein Receptors (BMPR) consisting of type I and type II serine/threonine kinase receptors to activate intracellular signaling molecules Smad1, Smad5 and Smad8 via serine phosphorylation [20].Type I receptors are classified as either BMPR-Ia receptors, which signal through Activin Receptor-like Kinases Alk2, Alk3 and Alk6, or BMPR-Ib receptors which utilize Alk4, Alk5 and Alk7 as signaling intermediates.

Other BMPs such as BMP2 have been shown to drive mesenchymal cell precursors into an osteoblast-specific differentiation pathway by increasing expression of runt-related transcription factor 2 (RUNX2)[1, 21], which may be a focal point for the integration of signaling pathways controlling osteoblast differentiation [22]. SOX9, which plays a role in bone and cartilage development, also functions downstream from BMP signaling [23]. Similarly, peroxisome proliferator-activated receptor $\gamma(\operatorname{PPAR} \gamma)$, the predominant regulator of adipocyte differentiation, is specifically activated by endogenous BMP4 in concert with CCAAT/enhancer-binding protein $\alpha(\mathrm{C} / \mathrm{ebp} \alpha)$ to drive adipocyte differentiation [24, 25].

The activity of BMPs may be modified by other factors to direct differentiation along different pathways. For example, BMP2 induces chondrogenesis whereas inhibitors of BMP2 promote tendon differentiation $[13,26]$. Antagonism between BMP and Fibroblast Growth Factor (FGF) signaling pathways regulates diversification of cartilage and tendon 
cells from a common precursor [27, 28]. Connective Tissue Growth Factor (CTGF) is a cysteine-rich secreted protein characterized in 1991 by several groups [29-31], which has been shown to negatively regulate BMP2-stimulated osteoblast differentiation [32]. CTGF belongs to the CCN gene family of early-growth response genes which regulate proliferation and differentiation of connective tissue cells [33, 34]. CTGF has been shown to interact directly with BMP2 [35], as well as BMP4 and TGF- $\beta 1$ [36] via the CR domain, a module also present in chordin and von Willebrand factor consisting of ten regularly spaced cysteine residues interspersed with other amino acids [37]. CTGF mRNA expression was increased in a chicken tendon injury model [38], suggesting a potential role in tendon repair. Treatment of mesenchymal stem cells with CTGF promoted differentiation into fibroblasts expressing Coll and tenascin-C [4].

Transplanted TSCs have been shown to promote tendon repair in a rat patellar tendon window defect model [39]. However, there is evidence that impaired differentiation of tenocytes may contribute to tendinopathy. For example, calcific deposits can been seen in a subset of tendinopathies called calcifying tendinopathy which may result from erroneous differentiation of TSCs to chondrocytes or osteoblasts [40]. Therefore, a more thorough understanding of the factors and environments which regulate differentiation of resident TSCs may provide therapeutic targets for pushing TSCs into a tenogenic differentiation pathway as a future treatment for tendinopathy.

The aim of our study was to examine the role BMP12 plays in the differentiation of TSCs isolated from rat tendon and to determine if CTGF was required for tenogenic differentiation. Our results showed that BMP12 stimulated expression of tenocyte differentiation markers and that CTGF promoted this effect via the Smad1/5/8 signaling pathway. Knockdown of CTGF expression diminished tenocyte differentiation. We further provide evidence for the direct interaction of CTGF and BMP12 involving the CR domain of CTGF, similar to its interaction with other BMP molecules. These results suggest that CTGF may be an appropriate therapeutic tool for future treatment of tendinopathy.

\section{Materials and Methods}

Isolation and culture of rat TSCS

Experiments were performed in accordance with the National Institutes of Health Guidelines for the Care and Use of Laboratory Animals. Animal care procedures were reviewed and approved by the Animal Research Ethics Committee, Third Military Medical University, China. 8-week-old Sprague Dawley rats weighing 250-300 g were used were housed under a $12 \mathrm{~h}$ light/dark cycle in a pathogen-free area with free access to water and food. Isolation and culture of rat TSCs were performed as previously described $[1,6]$. Briefly, intact flexor digitorum longus tendons from both hind feet were dissected after euthanasia. Midsubstance tissue was minced in sterile phosphate-buffer saline (PBS) and digested in $3 \mathrm{mg} / \mathrm{mL}$ of type I collagenase (sigma-Aldrich, St.Louis, MO, USA), $2.5 \mathrm{~h}$ at $37^{\circ} \mathrm{C}$. A $70 \mathrm{~mm}$ cell strainer was used to prepare a single cell suspension. The cells were washed in PBS and centrifuged at 300xg for $5 \mathrm{~min}$, then resuspended inDulbecco's modified Eagle's medium (DMEM)( Gibco, Carlsbad, CA,USA) with 10\% fetal bovine serum, $100 \mathrm{U} / \mathrm{mL}$ penicillin, $100 \mathrm{mg} / \mathrm{mL}$ streptomycin and $2 \mathrm{mM}$ L-glutamine (all from Invitrogen, Carlsbad, CA, USA). Increasing dilutions of cells were plated and grown for $2 \mathrm{~d}$ at $37^{\circ} \mathrm{C}$ in $5 \% \mathrm{CO}_{2}$, then washed twice in PBS to remove nonadherent cells. On day 7 of culture, the cells were trypsinized with trypsin-EDTA solution (sigma-Aldrich), mixed together and cultured as passage 0 cells. Cells from passages 1-3 were used in all experiments.

To examine the dose-response curve for the effect of BMP12 on differentiation of TSCs, $6 \times 10^{4}$ cells were seeded on 6 well plates and cultured with $0,1,10,50$ or $100 \mathrm{ng} / \mathrm{ml}$ of BMP12 for $14 \mathrm{~d}$. Optimal dosage duration was determined by culturing TSCs in $50 \mathrm{ng} / \mathrm{mL}$ of BMP12 for $0 \mathrm{~d}, 3 \mathrm{~d}, 7 \mathrm{~d}, 14 \mathrm{~d}$. Each experiment was repeated a minimum of three times. These experiments indicated that the differentiation of TSCs was maximal when cells were grown in $50 \mathrm{ng} / \mathrm{mL}$ BMP12 for $14 \mathrm{~d}$, so this dosage and duration were utilized for remaining experiments. To assess receptor activation by BMP12, cells were exposed to LDN-193198 (4-[6-(4-piperazin-1-ylphenyl) pyrazolo[1,5-a]pyrimidin-3-yl]quinoline)(Selleckchem, Munich, Germany),

\section{KARGER}


a specific inhibitor of BMPR-Ia receptors Alk2, Alk3 and Alk6. Activation of BMPR-Ib receptors was examined by exposure of cells to 2-(5-benzo[1,3]dioxol-5-yl-2-tert-butyl-3H-imidazol-4-yl)-6-methylpyridine hydrochloride (SB-505124)(Santa Cruz Biotechnology, Santa Cruz, CA, USA), which specifically inhibits Alk4, Alk5 and Alk7.

\section{ELISA}

TSCs were isolated as previously described and $5 \times 10^{3}$ cells were seeded on 12 well plates and grown in the presence of 0 or $50 \mathrm{ng} / \mathrm{mL}$ BMP12 for $0 \mathrm{~d}, 3 \mathrm{~d}, 7 \mathrm{~d}, 14 \mathrm{~d}$. Quantitation of ColI in TSC cells was determined by ELISA using commercial kits purchased from Chondrex (Redman, WA, USA). Coll was solubilized by lysis of cells in $0.5 \mathrm{M}$ acetic acid. Results were expressed in $\mu \mathrm{g}$ protein per $\mu \mathrm{g}$ DNA isolated (DNA was isolated by Quiagen DNA extraction kit following the manufacturer's instructions )from the same cells.

\section{CTGF construction and transfection}

Total RNA was extracted from rat TSCs, and CTGF (NCBI Reference Sequence NM_001901.2) cDNA and negative control sequence were cloned and amplified by RT-PCR using primers 5'-ATGACCGCCGCCAGTAT-3', 5'-AGACAGTGAAGCCGAGGG-3' and 5'-AATGACAACGCCTCCTGC-3', 5'-ACACCTCATACATGGCTGCC-3', respectively. The amplicons were then subcloned into a pcDNA3/V5-His TOPO TA vector (Invitrogen) and transfected into rat TSCs, using the TransFast transfection reagent (Promega) according to the manufacturer's instructions. Twenty-four hours after transfection G418 (Life Technologies, Carlsbad, CA, USA) was added to $500 \mathrm{mg} / \mathrm{ml}$ for stable transfectant selection. The cell transfected with empty vector as control.

\section{CTGF shRNA and transfection}

To generate a shRNA-expression plasmid, we designed a shRNA sequence and one scrambled sequence according to the CTGF nucleotide sequence in GenBank. The targeted nucleotide seguence was 5'-GCCCAGACCCAACTATGATTA-3' (nucleotides 372-392), and they were designed and synthesized by RiboBio (Guangzhou, China). The shRNA sequence was inserted into the pLKO.1 lentiviral plasmid vector (Sigma-Aldrich, St. Louis, MO, USA) and lentiviral particles were produced by transient transfection of HEK 293T cells. To incorporate the CTGF shRNA, TSCs were incubated with infectious lentivirus medium for 36h. The empty vector pLKO.1 or pLKO.1 vector containing scrambled shRNA served as negative controls.

\section{qRT-PCR}

$2 \times 10^{6}$ TSCs were isolated and grown in 6 well plates as previously described in the presence of drugs and/or lentiviral particles. At the indicated time points, cells were harvested for analysis of mRNA expression by qRT-PCR. RNA was extracted using an RNeasy mini kit (Quiagen, GmbH, Hilden, Germany). Reverse transcription of cDNA was performed using a First Strand cDNA kit (Promega, Madison, WI, USA). PCR of 5 $\mu \mathrm{L}$ total cDNA from each sample was carried out in $25 \mu \mathrm{L}$ reactions containing Platinum SYBR Green qPCR SuperMix-UDG and appropriate primers in the ABI StepOne Plus System from Applied Biosystems (Foster City, CA, USA). Primers in Table 1 were used. Cycling parameters were as follows: Denaturation, $95^{\circ} \mathrm{C}$ for 10 min followed by 45 cycles consisting of denaturation at $95^{\circ} \mathrm{C}$ for $20 \mathrm{~s}$, annealing at temperature $60^{\circ} \mathrm{C}$ for $25 \mathrm{~s}$, extension at $72^{\circ} \mathrm{C}$ for $30 \mathrm{~s}$, and controlled rise to $95^{\circ} \mathrm{C}$ with a heating rate of $0.1^{\circ} \mathrm{C} / \mathrm{s}$. Target gene expression was normalized to that of $\beta$-actin. Relative gene expression was calculated using the $2^{-\Delta \Delta C T}$ formula.

\section{Immunofluorescence staining}

Expression of tenogenic markers Scx and Tnmd was examined by immunofluorescence staining. Cultured rat TSCs were fixed with 4\% paraformaldehyde in PBS. Cells were washed 3 times in PBS, permeabilized with $0.5 \%$ Triton X-100 in PBS, and blocked with 5\% normal donkey serum (Jackson ImmunoResearch, West Grove, PA, USA) in PBS containing 0.1\% Triton X-100 (PBST). Immunostaining was performed overnight at $4^{\circ} \mathrm{C}$ using either rabbit anti-human Scx (1:100)(Abcam) and rabbit anti-human Tnmd (1:100) obtained from Santa Cruz Biotechnology. The cells were washed 3 times in PBST, incubated for $1 \mathrm{~h}$ at room temperature with Cy3-conjugated goat anti-rabbit IgG $(\mathrm{H}+\mathrm{L})$ from Abcam, and Cell nuclei were counterstained with DAPI (Sigma). Observed and photographed under a fluorescence microscope (Leica, Wetzlar, Germany)

\section{KARGER}




\section{Western blot}

Western blots were performed according to Safadi et al. [41]. Protein was extracted from rat TSC cultures with Protein Extraction Kit (Pierce) at the appropriate time points. Protein samples were mixed with an equal volume of $2 \times$ SDS sample buffer, boiled for $10 \mathrm{~min}$ and separated by $7.5 \%$ SDS-PAGE. The gels were transferred to nitrocellulose using a semi-dry transfer apparatus. Blots were blocked for $2 \mathrm{~h}$ in $5 \%$ non-fat dry milk dissolved in Trisbuffered saline containing $0.2 \%$ Tween-20 (TBST), then incubated in primary antibodies overnight at $4^{\circ} \mathrm{C}$. The following antibodies were used: Anti-CTGF (1:5000), anti-BMP12 (1:1000), anti-Scx (1:500), anti-Tnmd (1:50), anti-ColI (1:5000), and anti-Tn-C (1:2000), all purchased from Abcam (Cambridge, MA, USA); anti-Smad1/5/8 (1:200), anti-phosphoSmad1/5/8 (1:1000), and anti-Smad 1 (1:200), purchased from Santa Cruz Biotechnology. Following primary antibody incubation, blots were washed 3times in TBST and incubated in goat anti-rabbit IgG (H\&L)-HRP conjugate (1:2000) $2 \mathrm{~h}$ at room temperature. Signals were visualized using an ECL detection kit (Pierce, Rockford, IL, USA)

\section{Chemical crosslinking assay}

Chemical crosslinking was performed using the amine reactive crosslinker dithiobis (succinimidyl) propionate (DSP; Pierce Biotechnology, Rockford, IL). Anti-BMP12, recombinant BMP12 and recombinant CTGF were purchased from Abcam. Recombinant
Table 1. Primer sequences of target genes for realtime PCR assays

\begin{tabular}{|c|c|c|}
\hline Gene & & Primer Sequence \\
\hline \multirow[t]{2}{*}{ CTGF } & forward & 5'- CTCGCCTTGGTGCTCCTCCT-3' \\
\hline & reverse & 5'- CTACTCGGGTTCCTGGCGTG-3' \\
\hline \multirow[t]{2}{*}{ Scx } & forward & 5'- CGTGCTACTGGTGGGTGA-3' \\
\hline & reverse & 5'-GTGGAAGACGGAGTCGTT-3' \\
\hline \multirow[t]{2}{*}{ Tnmd } & forward & 5'- CAGGACTTTGAGGAGGATGG-3' \\
\hline & reverse & 5'-CGTGGTCTGTTCGTTCGCTC-3' \\
\hline \multirow[t]{2}{*}{ ColI } & forward & 5'-CTCAGCCCTCTGTGCCT-3' \\
\hline & reverse & 5'-CTCATACCTTCGCTTCCAA-3' \\
\hline \multirow[t]{2}{*}{ Tn-C } & forward & 5'-TCTCCGAAGGAAATCAT-3' \\
\hline & reverse & 5'-TAATGGGCGTGTCACAG-3' \\
\hline \multirow[t]{2}{*}{ BMPRIa } & forward & 5'-GCCATTGCTTTGCCATTAT-3' \\
\hline & reverse & 5'-ACTACCGTCGCAGGCTACC-3' \\
\hline \multirow[t]{2}{*}{ BMPRIb } & forward & 5'-ATGGGAAAGTGGCGTGGAGA-3' \\
\hline & reverse & 5'-GGATGAGCAGACAGTCGCCG-3' \\
\hline \multirow[t]{2}{*}{ Runx2 } & forward & 5'-CССААСТTССТGTGCTCC-3' \\
\hline & reverse & 5'-CTGCTCCGTTCTCAAAGTGA-3' \\
\hline \multirow[t]{2}{*}{ Sox9 } & forward & 5'-GCACATCAAGACGGAGCAA-3' \\
\hline & reverse & 5'-CCGAGATGAGGTGGAAGTGGA-3' \\
\hline \multirow[t]{2}{*}{ PPAR $\gamma$} & forward & 5'-TCAGGTTTGGGCGAATG-3' \\
\hline & reverse & 5'-GGAAGGGCGACTGGTTT-3' \\
\hline
\end{tabular}

BMP12 at $2 \mathrm{nM}$ was incubated with recombinant CTGF or the constructs CTGF- $\delta$ CR (mRNA encoding CTGF lacking the CR domain, CTGF-CT (the C terminus of CTGF) and CTGF-CR (a construct encoding a secreted form of only the CR domain of CTGF) in a volume of $10 \mathrm{nM}$ for $1 \mathrm{hr}$ at room temperature. DSP was added to a final concentration of $0.15 \mathrm{mM}$, and the mixture was incubated for $15 \mathrm{~min}$ at room temperature. The reaction was terminated with the addition of Tris- $\mathrm{HCl} \mathrm{pH} 7.4$ at a final concentration of $30 \mathrm{mM}$. Samples were electrophoresed on non-reducing SDS gels and probed with anti-BMP12 at 1:1000 as previously described.

\section{Statistical analysis}

Data shown are representative of at least 3 independent experiments. All results are expressed as mean \pm SD. The association among the variables was determined by two-way ANOVA with Fisher's PLSD post hoc test using StatView 5.0 (SAS Institute Inc., Cary, NC, USA), and Statistical analyses were performed using SPSS software (version 17.0, SPSS Inc., Chicago, IL). p<0.05 was considered statistically significant.

\section{Results}

BMP12 stimulates expression of tenogenic markers in rat TSCs

To examine the effect of BMP12 on tenogenesis, we isolated TSCs from rat flexor digitorum longus tendons from both hind feet and exposed them to increasing concentrations of BMP12 (1 -100 ng/mL) for $3 \mathrm{~d}, 7 \mathrm{~d}$ and $14 \mathrm{~d}$. Expression of Scx, Tnmd, ColI and Tn-C mRNA increased in a dose-related fashion (Fig. 1A), peaking at $50 \mathrm{ng} / \mathrm{mL}$ for Scx at each exposure time. Tnmd transcript levels were maximal at $50 \mathrm{ng} / \mathrm{mL}$ for $3 \mathrm{~d}, 7 \mathrm{~d}$ and $14 \mathrm{~d}$ exposures, but did not increase significantly during $14 \mathrm{~d}$ exposures. ColI mRNA peaked at $50 \mathrm{ng} / \mathrm{mL}$ for $3 \mathrm{~d}$ 
Fig. 1. BMP 12 stimulates expression of tenocyte lineage markers. Rat TSCs were isolated and treated with BMP12 for dose-response and time course studies. A: Expression of Scx, Tnmd, ColI, and Tn-C mRNA quantified by qRTPCR after treatment with increasing BMP12 concentrations $(0,1,10,50$, and $100 \mathrm{ng} / \mathrm{ml}$ ) for $3 \mathrm{~d}, 7 \mathrm{~d}$, and 14 d. Fold change in mRNA level is expressed relative to the control group. B: Scx, Tnmd, Coll, and Tn-C protein expression by western blot after treatment with $50 \mathrm{ng} / \mathrm{ml} \mathrm{BMP12}$ for 0 d, 3 d, 7 d, 14 d. Protein expression is normalized to the level of $\beta$-actin. C: Elisa analysis of ColI and $\mathrm{Tn}-\mathrm{C}$ after treatment with $50 \mathrm{ng} / \mathrm{mL}$ BMP12 for $0 \mathrm{~d}, 3 \mathrm{~d}, 7$ $\mathrm{d}, 14 \mathrm{~d}$. Coll is quan-
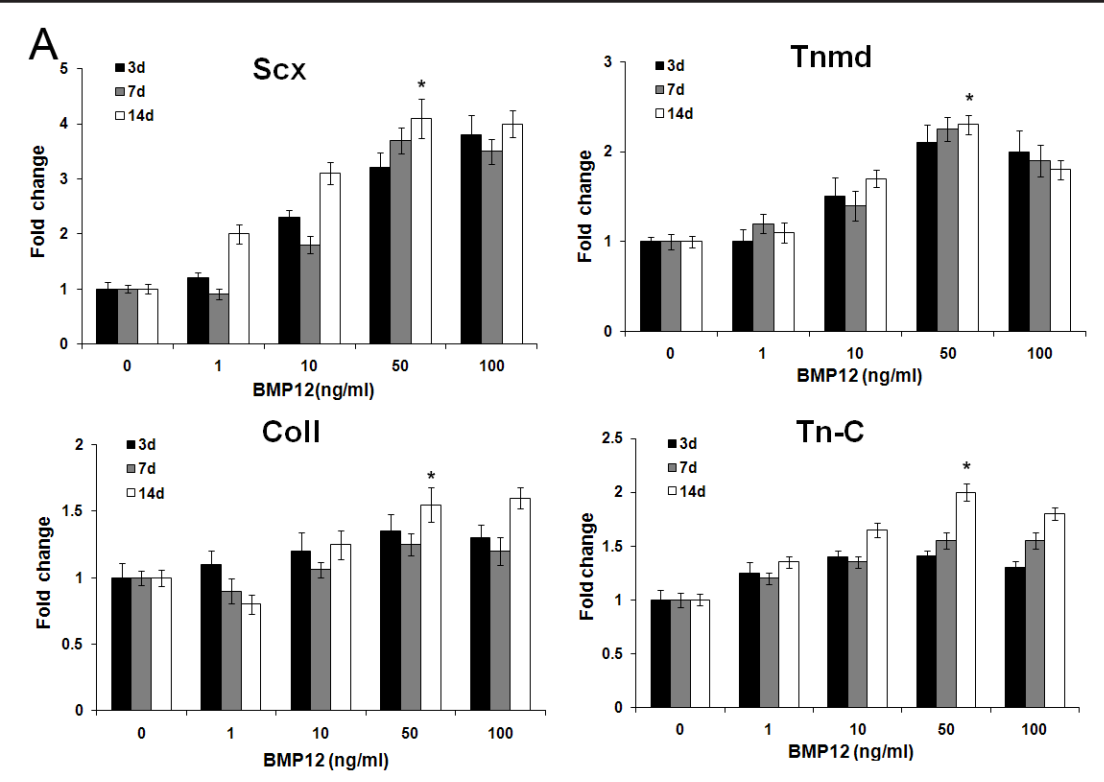

B
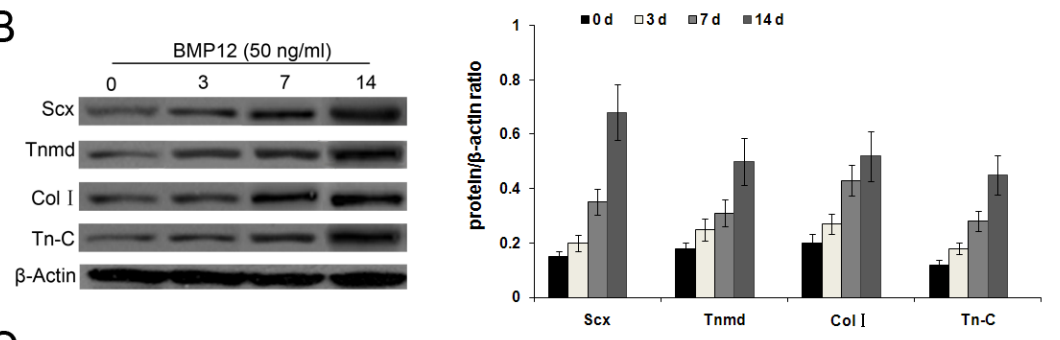

C
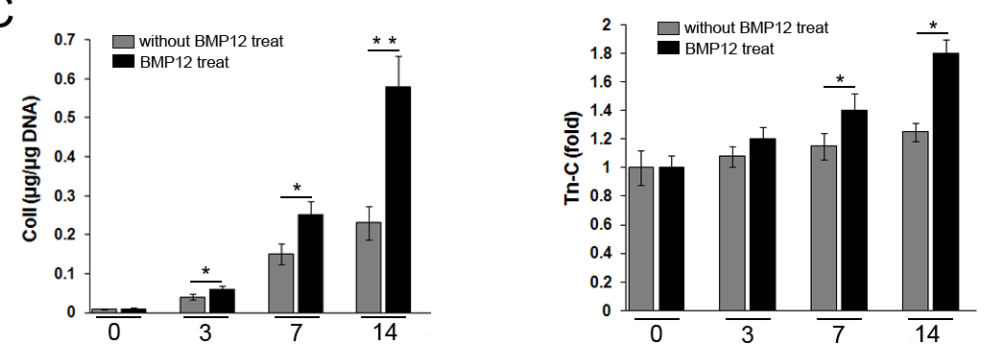

tified in $\mu \mathrm{g}$ protein and normalized to total DNA. Tn-C is expressed as fold change relative to the control groups. ${ }^{*} \mathrm{p}<0.05,{ }^{* *} \mathrm{p}<0.01$ compared to the control group.

and $14 \mathrm{~d}$ exposures, but was only modestly increased at $50 \mathrm{ng} / \mathrm{mL}$ for $7 \mathrm{~d}$ exposures. Tn-C peaked at $50 \mathrm{ng} / \mathrm{mL}$ for $7 \mathrm{~d}$ and $14 \mathrm{~d}$ exposures, and increase significantly over $1 \mathrm{ng} / \mathrm{mL}$ for 3 d exposure.

To confirm our qRT-PCR results, we examined protein expression of the above markers at progressively longer exposures to $50 \mathrm{ng} / \mathrm{mL}$ BMP12 using western blots. Protein levels were quantified relative to $\beta$-actin expression (Fig. 1B). This experiment confirmed our mRNA results and demonstrated increasing expression of Scx, Tnmd, ColI and Tn-C protein over time, with peak expression after $14 \mathrm{~d}$ of BMP12 exposure. We extended these results by ELISA analysis of the levels of type I collagen and Tn-C in our cultures exposed to 50 ng/mL BMP12 (Fig. 1C). These results showed that increasing levels of ColI in TSC cultures over time were further enhanced after $7 \mathrm{~d}$ or more exposure to BMP12, compared to nonexposed control cells. Similarly, modest increases in Tn-C over time in control cells were further enhanced by BMP12 exposure for $7 \mathrm{~d}$ or more. These results together demonstrate that BMP12 stimulates markers of tenogenesis in rat TSC cultures. 
Fig. 2. CTGF stimulates expression of tenocyte lineage markers. Rat TSCs were transfected with vector containing CTGF plasmid (CTGFTSC) or negative control plasmid (NC-TSC), the empty vector served as control. A: CTGF protein expression shown on western blot after transfection with vectors carrying the CTGF plasmid. Expression is normalized to the levels of $\beta$-actin. B: Scx, Tnmd, ColI, and Tn-C mRNA expression analyzed by qRT-PCR after transfection with CTGF-TSC or NC-TSC, with and without BMP12 exposure at $50 \mathrm{ng} / \mathrm{ml}$ for $14 \mathrm{~d}$. mRNA is expressed relative to the non-transfected control group. ${ }^{*} \mathrm{p}<0.05$, ${ }^{* *} \mathrm{p}<0.01$ compared to the control group. C: Representative images of immunofluorescence staining of Scx and Tnmd after transfection with CTGF-TSC or NC-TSC, with and without BMP12 exposure at $50 \mathrm{ng} / \mathrm{ml}$ for $14 \mathrm{~d}$. $(200 \times)$.

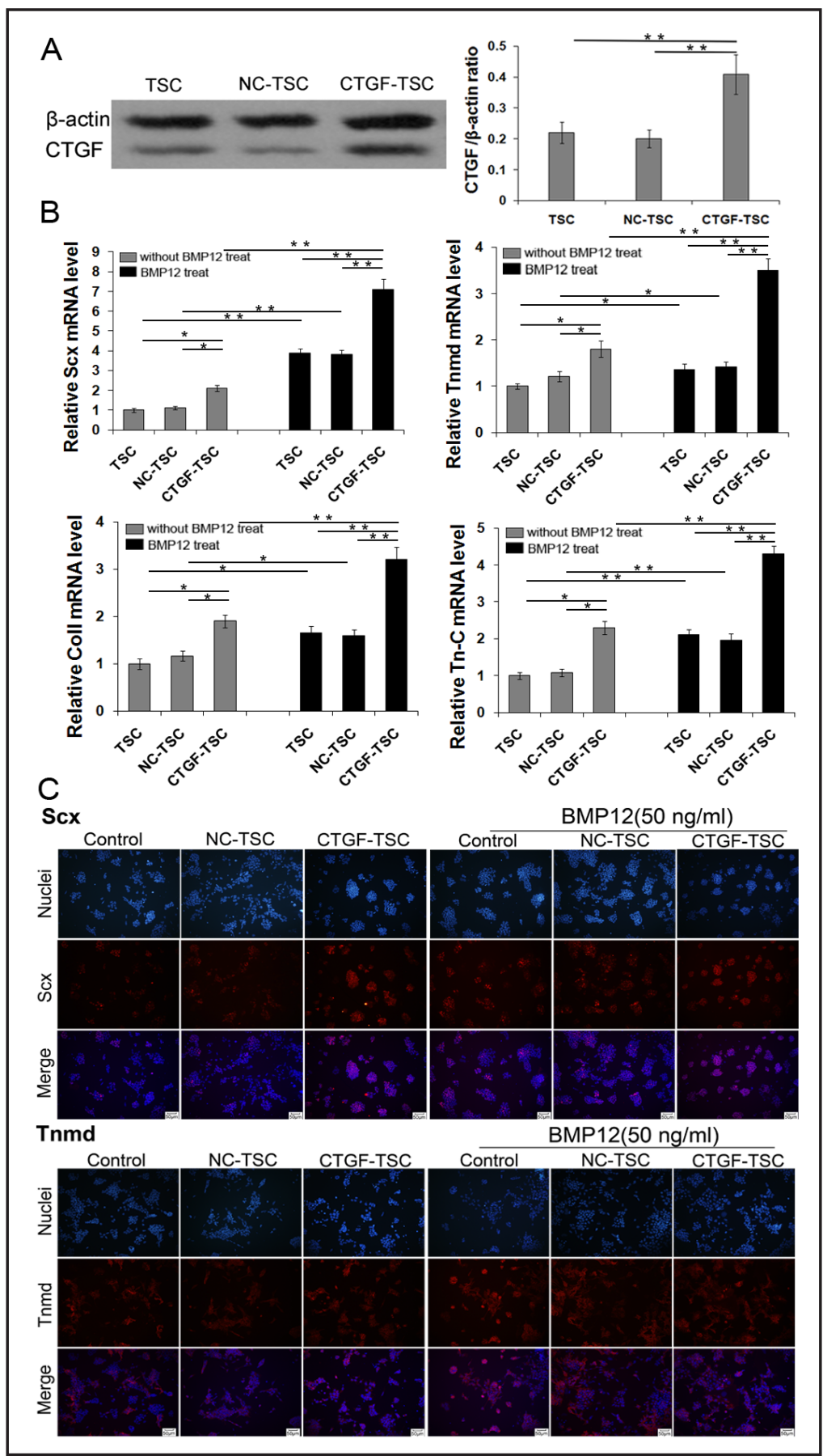

CTGF overexpression enhances tenogenesis in the presence and absence of BMP12

We next examined the effect of CTGF exposure on tenogenesis in the presence and absence of BMP12. To accomplish this, we used a vector carrying a human CTGF gene in to overexpress CTGF in rat TSCs and compared expression of Scx, Tnmd, ColI and Tn-C to cells transfected with empty vector or the same vector carrying scrambled sequence. We first confirmed by western blot that transfection with the CTGF vector increased CTGF levels relative to $\beta$-actin expression 2-fold compared to TSCs injected with the negative control vector (Fig. 2A). We then quantified by qRT-PCR mRNA levels of Scx, Tnmd, Coll and Tn-C in rat TSC cultures transfected with the CTGF and negative control vectors and grown in the presence or absence of $50 \mathrm{ng} / \mathrm{mL}$ BMP12 for $14 \mathrm{~d}$. CTGF over-expression in the absence of BMP12 increased 
Fig. 3. The effect of CTGF on expression of non-tenocyte lineage markers. Expression of PPARg, RUNX2, and SOX9 mRNA quantified by qRT-PCR after transfection with CTGF-TSC or NC-TSC, with and without BMP12 exposure at $50 \mathrm{ng} / \mathrm{ml}$ for $14 \mathrm{~d}$. Fold change expression is relative to the non-transfected control group. D: p-Smad1/5/8 and total Smad1visualized by Western blot after transfection with CTGF-TSC or NC-TSC, with and without BMP12 exposure at $50 \mathrm{ng} / \mathrm{ml}$ for $14 \mathrm{~d} .{ }^{*} \mathrm{p}<0.05,{ }^{* *} \mathrm{p}<0.01$ compared to the non-transfected control group.

transcript levels of these markers after 14 $\mathrm{d}$ of culture compared to non-transfected cells or to cells transfected with the negative control vector. Exposure to BMP12 for $14 \mathrm{~d}$ stimulated expression of Scx, Tnmd, Coll and Tn-C mRNA in both non-transfected TSCs and in cells transfected with the negative control vector, and CTGF over-expression in conjunction with BMP12 exposure further enhanced expression of these markers from 1.9- to 2.5-fold. We confirmed these results by immunofluorescence assays showing increased expression of Scx and Tnmd protein in rat TSCs $14 \mathrm{~d}$ after infection with the CTGF vector compared to nontransfected cells or cells transfected with the negative control vector. The effect of CTGF on

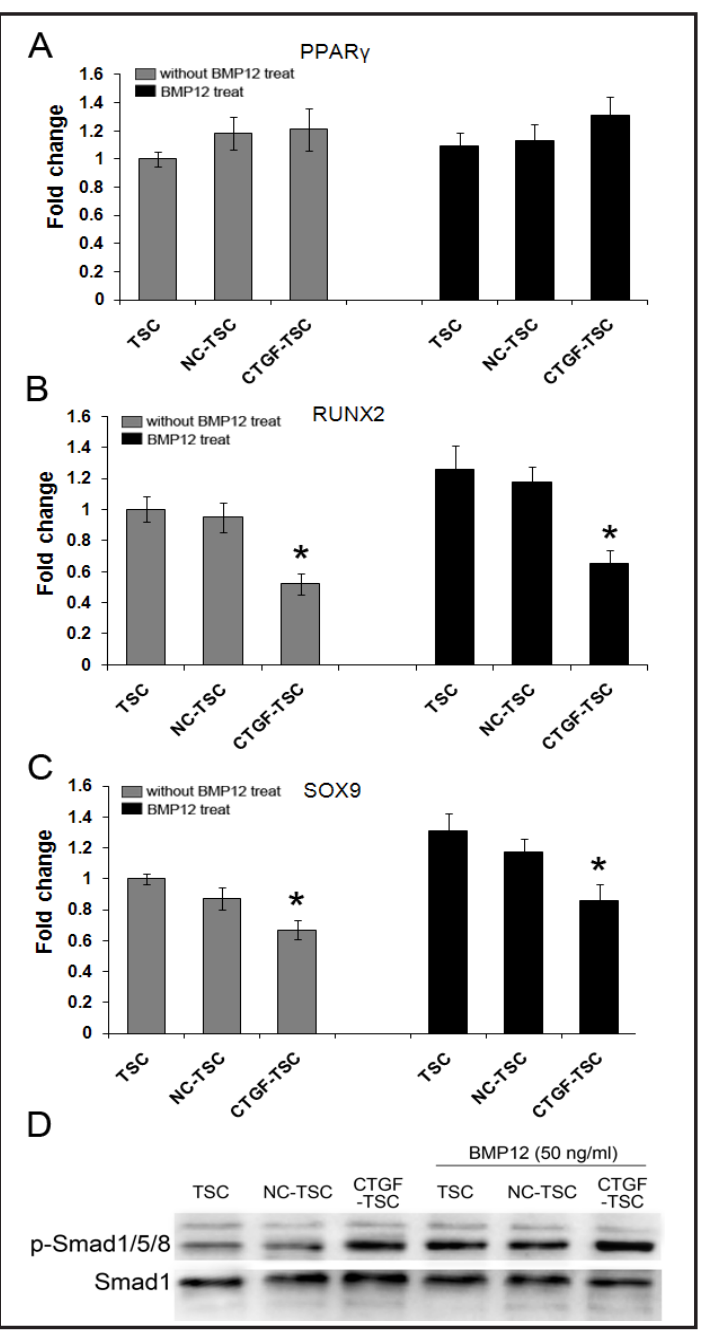

Scx and Tnmd expression was similar in the presence and absence of BMP12. These results demonstrate that CTGF promotes the tenogenic effect of BMP12 on rat TSCs.

CTGF acts via the Smad 1/5/8 pathway to promote tenogenesis and inhibit markers of adipocyte and osteoblast differentiation

Disturbances in the normal differentiation of tenocytes may result in the development of inappropriate cell types that impair the formation of tendon tissue following injury [40]. To determine if overexpression of CTGF directs the development of other cellular phenotypes, we examined the expression of adipocyte, osteoblast and chondrocyte markers following overexpression of CTGF in rat TSCs in the presence and absence of $50 \mathrm{ng} / \mathrm{mL}$ BMP12. CTGF had no significant effect on levels of the adipocyte marker PPAR $\gamma$ (Fig. 3A), either in the presence or absence of BMP12, nor did BMP12 exposure on control cells affect PPAR $\gamma$ expression. In contrast, CTGF overexpression significantly decreased levels of osteoblast-specific protein RUNX2 as well as SOX9, a chondrocyte marker (Figs. 3B and 3C, respectively), whether or not BMP12 was present. BMP12 exposure alone produced modest non-significant increases in RUNX2 and SOX9 in control cells, whereas CTGF overexpression appeared to downregulate this response to BMP12. Thus, CTGF may act to promote tenogenesis while simultaneously discouraging differentiation of bone and cartilage cell types.

Both BMP12 and CTGF appear to exert their effects via Smad1/5/8 pathways. When we examined Smad1/5/8 phosphorylation in control and CTGF-overexpressing rat TSCs, we observed that increased Smad activation stimulated by BMP12 alone was enhanced in cells also expressing CTGF (Fig. 3D). CTGF expression alone had a less prominent effect on 


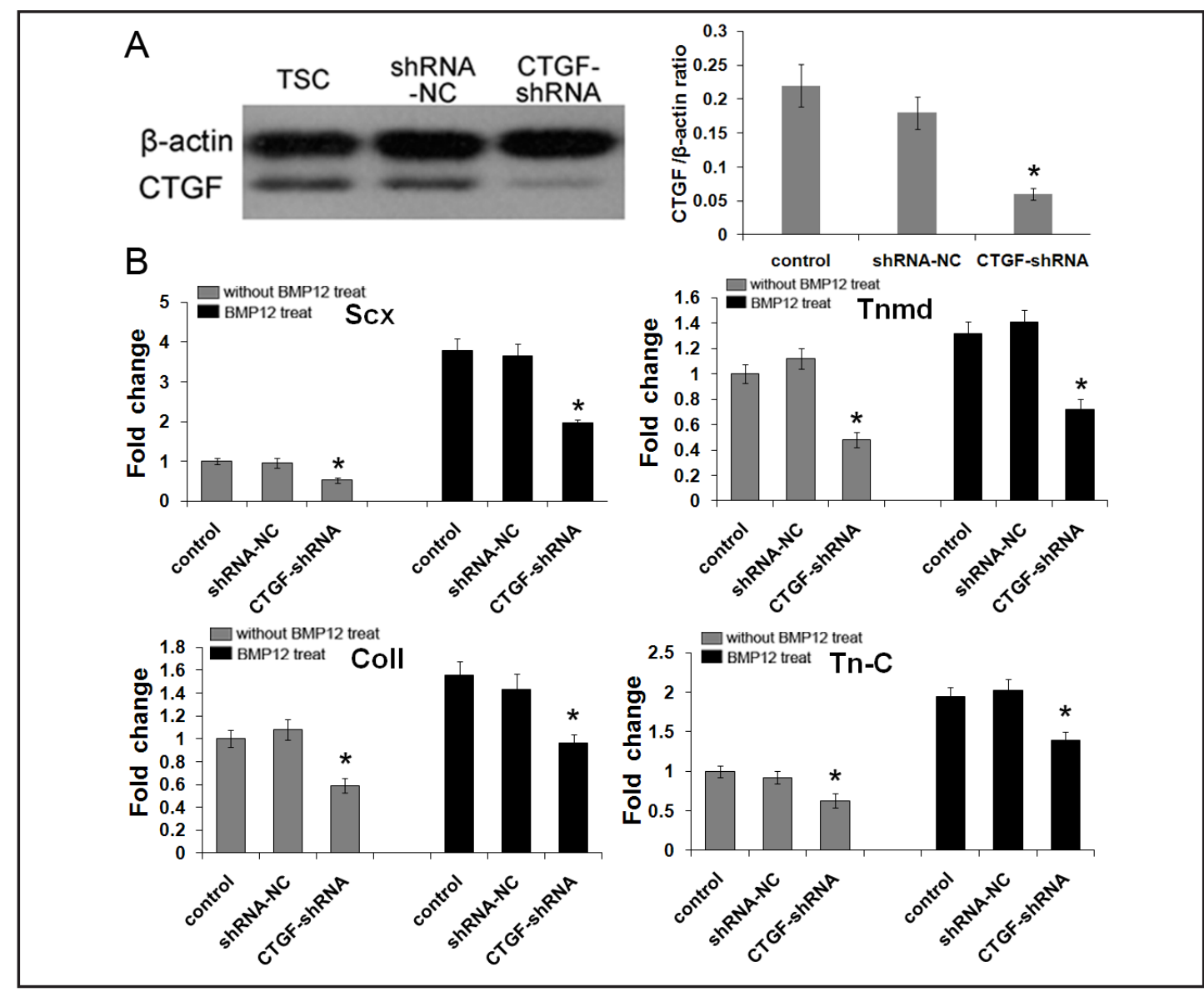

Fig. 4. CTGF knockdown decreases expression of tenocyte lineage markers. CTGF expression in rat TSCs was knocked down by infection with a lentiviral vector carrying shRNA targeting CTGF (CTGF-shRNA). The empty vector pLKO.1 or scrambled shRNA in pLKO.1 served as negative controls (shRNA-NC). A: CTGF protein expression after knockdown detected by western blot. $\beta$-actin served as loading control. Quantification of CTGF protein levels was performed by densitometry and normalized to the level of $\beta$-actin. B: Scx, Tnmd, ColI, and Tn-C mRNA expression quantified by qRT-PCR after transfection with shRNA-NC and CTGF-shRNA, with and without BMP12 exposure at $50 \mathrm{ng} / \mathrm{ml}$ for $14 \mathrm{~d}$. mRNA is expressed as fold change relative to the non-transfected control group. ${ }^{*} \mathrm{p}<0.05$.

Smad1/5/8 phosphorylation. Expression of Smad1 protein showed little change in these cells. It thus appears that CTGF and BMP12 work in concert via Smad activation to regulate tenogenesis.

\section{CTGF knockdown decreases expression of tenogenic markers in the presence and absence} of BMP12

To further examine the role of CTGF in tenocyte differentiation, we designed shRNA targeting CTGF expression and used the lentiviral vector pLKO.1 to incorporate the plasmid into the genome of rat TSCs. To show that ectopic incorporation of the plasmid did not affect CTGF expression, we also infected control rat TSCs with either the empty pLKO.1 vector or with the vector containing a scrambled shRNA. Fig. 4A demonstrates that in rat TSCs infected with the CTGF shRNA, CTGF expression normalized to $\beta$-actin was diminished approximately 3-4-fold with respect to non-infected control cells or cells infected with the nonsense vector, showing the effectiveness of our CTGF shRNA in knocking down CTGF expression. We then utilized our CTGF shRNA to knock down CTGF expression in rat TSCs grown in the presence and absence of $50 \mathrm{ng} / \mathrm{mL}$ BMP12 for $14 \mathrm{~d}$ and examined mRNA expression of tenogenic 


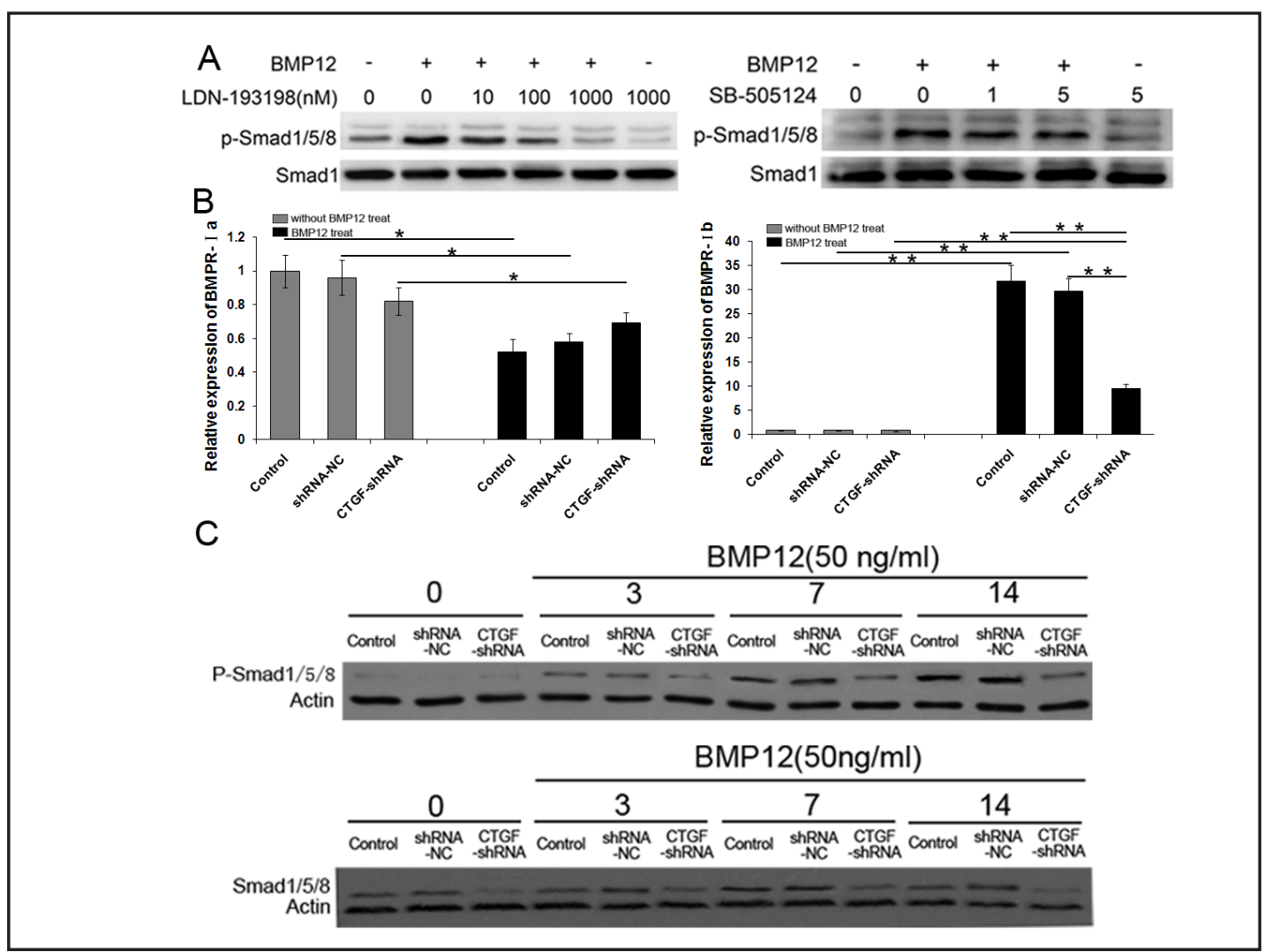

Fig. 5. BMP12 signaling is transduced by BMPR-Ia receptors. A: BMP12-induced phosphorylation of Smad1/5/8 after inhibition of Alk2, Alk3, and Alk6 by LDN-193189, and after inhibition of Alk4, Alk5, and Alk7 by SB-505124. Proteins detected by western blot. B: BMPR-Ia and BMPR-Ib mRNA expression quantified by qRT-PCR after transfection with shRNA-NC and CTGF-shRNA, with and without BMP12 exposure at $50 \mathrm{ng} / \mathrm{ml}$ for $14 \mathrm{~d}$. mRNA expressed relative to non-transfected control group without BMP12 treatment. ${ }^{* *} \mathrm{p}<0.01$ compared to the control group. C: $\mathrm{p}-\mathrm{Smad} 1 / 5 / 8$ (upper panel) and total Smad1/5/8 (lower panel) visualized by western blot after transfection with shRNA-NC and CTGF-shRNA, without and with BMP12 exposure at $50 \mathrm{ng} / \mathrm{ml}$ for $3 \mathrm{~d}, 7 \mathrm{~d}$, and $14 \mathrm{~d}$.

markers Scx, Tnmd, ColI and Tn-C. Knockdown of CTGF in the absence of BMP12 significantly reduced expression of Tnmd, ColI and Tn-C transcripts compared to non-infected cells or to cells infected with the nonsense vector $(p<0.05)$. Scx mRNA was non-significantly reduced approximately 2-fold. BMP12 stimulated expression of each marker mRNA in control or nonsense-vector infected cells, but expression of the CTGF shRNA significantly decreased marker expression to below control levels $(p<0.05)$. These results provide further evidence that CTGF expression facilitates differentiation of tenocytes from TSCs.

\section{BMP12 signaling is mediated by BMPR-Ia}

The previous results have shown that BMP12 and CTFG signaling are both mediated through the same Smad1/5/8 pathway. BMP12 signaling is conveyed through heteromeric complexes of type I and type II receptors. We used inhibitors of BMPR-Ia (LDN-193198) and BMPR-Ib (SB-505124) to test the receptor pathways which mediate the response to BMP12. We exposed rat TSCs to these inhibitors in the presence and absence of $50 \mathrm{ng} / \mathrm{mL}$ BMP12 for $14 \mathrm{~d}$ and compared Smad1/5/8 phosphorylation in western blots to non-exposed control cells (Fig. 5A). BMP12 stimulated phosphorylation of Smad1/5/8 which was inhibited by LDN-193198 in a dose-responsive fashion. In contrast, SB-505124 failed to alter Smad1/5/8 activation. Expression of Smad1 was not affected by these treatments. These results indicate that Smad activation by BMP12 is mediated by BMPR-Ia receptors. 
Fig. 6. Interaction between BMP12 and CTGF in vitro. BMP12 was crosslinked to full-length CTGF (lane2), CTGF lacking the CR domain (CTGF$\delta \mathrm{CR}$; lane 3), the CT domain (CTGF-CT; lane 4) and the CR domain (CTGFCR; lane 5). Crosslinked products were detected by western blot probed with anti-BMP12. Bands corresponding to BMP12 dimer, BMP12-CTGF complex (B:C) and BMP12-CTGF-CR complex (B:CR) are shown.

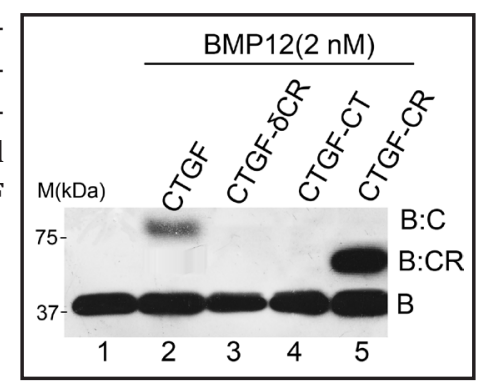

To examine the effect of CTGF on expression of BMPR-Ia and BMPR-Ib, we used qRTPCR and examined receptor expression in rat TSCs infected with the control or CTGF shRNA lentiviral constructs in the presence and absence of BMP12 as described above. Exposure to BMP12 for $14 \mathrm{~d}$ produced a significant decrease in BMPR-Ia expression in both infected and non-infected cells (Fig. 5B, left panel). Possibly prolonged exposure exerted downregulatory pressure on BMPR-Ia expression. Nonetheless, these results clearly demonstrate that enhanced activation of Smad proteins was not the result of increased expression of BMPR-Ia receptors and further shows that CTGF knockdown does not impact expression of this receptor in either the presence or absence of BMP12. In contrast, relative expression of BMPR-Ib was strongly enhanced by BMP12 in non-infected and control cells (Fig. 5B, right panel). Despite upregulation of BMPR-Ib by BMP12, SB-505124 inhibition failed to diminish Smad phosphorylation, suggesting that BMPR-Ib does not mediate signaling by BMP12 that impacts tenocyte differentiation. On the other hand, knockdown of CTGF expression by shRNA significantly suppressed the increase in BMPR-Ib expression induced by BMP12, compared to BMP12-exposed control cells ( $\mathrm{p}<0.01$; Fig. 5B, right panel). Thus, CTGF may act in concert with BMP12 to upregulate and maintain BMPR-Ib expression.

Finally, we examined the effect of CTGF downregulation by shRNA infection on Smad1/5/8 expression and phosphorylation in rat TSCs exposed to $50 \mathrm{ng} / \mathrm{mL} \mathrm{BMP12}$ for progressively longer periods from $3 \mathrm{~d}$ to $14 \mathrm{~d}$. Using western blots, we show that progressively longer BMP12 exposures induced increasing Smad1/5/8 phosphorylation relative to $\beta$-actin expression, peaking at $14 \mathrm{~d}$ exposure (Fig. 5C, upper panel), but had only minimal impact on levels of total Smad1/5/8 protein (lower panel). At each BMP12 exposure time, knockdown of CTGF expression diminished Smad phosphorylation, and also modestly suppressed levels of total Smad1/5/8 protein, even in the absence of BMP12. Thus, it may be the case that CTGF controls Smad activation in part by maintaining levels of total Smad protein in rat TSCs.

\section{BMP12 interacts directly with CTGF in vitro}

In our final study, we demonstrate a physical interaction between BMP12 and CTGF in chemical crosslinking experiments. Recombinant BMP12 at $2 \mathrm{nM}$ was incubated with recombinant full-length CTGF, CTGF lacking the CR domain (CTGF- $\delta$ CR), the CT domain of CTGF (CTGF-CT) or the CR domain (CTGF-CR). Crosslinking was carried out with the amine linker dithiobis(succinimidyl)propionate and the products were examined by western blot probed with anti-BMP12. Results show an expected dimer of BMP12 present in all lanes, and higher molecular weight products representing the BMP12-CTGF complex (B:C), and BMP12 complexed to the CR domain (B:CR). Notably, BMP12 did not interact with a CTGF construct lacking the CR domain (CTGF- $\delta$ CR), nor did it bind the carboxy terminal cysteine knot (CT) domain, illustrating that the presence of the CR domain is sufficient for the interaction of BMP12 with CTGF. These results suggest that the physical association of CTGF with BMP12 may regulate the effect of BMP12 on tenocyte differentiation; however, the mechanism by which this interaction occurs remains to be determined.

\section{Discussion}

Our experimental aim in this research was to examine the role of BMP12 in driving tenogenesis using isolated rat TSCs to model tenocyte differentiation. Results show that 
BMP12 increased expression of tenogenic markers Scx, Tnmd, Tn-C and Coll in a doseand time-dependent manner. Overexpression of CTGF also stimulated expression of these markers, whereas use of a CTGF shRNA to knock down CTGF expression diminished their expression in the presence or absence of BMP12. These effects of BMP12 and CTGF were mediated by increased phosphorylation and activation of Smad1/5/8 proteins via type I BMPR-Ia receptors since an inhibitor of Alk2, Alk3 and Alk6 prevented Smad phosphorylation, while an inhibitor of BMPR-Ib receptors had no effect on Smad activation. Knockdown of CTGF expression also suppressed BMP12-induced Smad activation and reduced total Smad1/5/8 proteins. The effect of CTGF on tenogenic differentiation may involve a direct physical interaction of BMP12 with the CR domain of CTGF. We conclude that BMP12 plays a critical role in tenogenesis via the Smad1/5/8 pathway and that CTGF promotes the tenogenic effects of BMP12.

The factors which regulate and control tenogenesis are still poorly understood. BMPs including BMP12, 13 and 14 have been shown to induce formation of new connective tissue [17] and to enhance tendon repair in several tendon injury models [18, 19]. In our study using isolated rat TSCs, BMP12 treatment enhanced transcription of tenogenic lineage genes Scx and Tnmd, and increased expression of connective tissue-associated proteins Tn-C and ColI, consistent with facilitation of tendon development. Independent from BMP12, CTGF may also promote tendon development by directing differentiation of mesenchymal precursor cells [15]. Similarly, our results also showed that CTGF overexpression in rat TSCs stimulated tenocyte lineage markers in the absence of BMP12 and further enhanced their expression induced by BMP12. Thus, CTGF may play an important role in tendon development by acting in conjunction with BMP12. CTGF may be required for maximal BMP12 effectiveness since knockdown of CTGF expression by a CTGF-targeted shRNA reduced the BMP12-induced increases in tenocyte markers.

Disordered differentiation of tenocytes has been suggested to contribute to calcifying tendinopathy by erroneous differentiation of bone or cartilage cell types [40]. We asked if overexpression of CTGF in rat TSCs could induce other cellular phenotypes by examining expression of PPAR $\gamma$, RUNX2 and SOX9 as differentiation markers for adipocytes, osteoblasts and chondrocytes, respectively. CTGF had little effect on PPAR $\gamma$, in the presence or absence of BMP12, but did suppress expression of RUNX2 and SOX9 regardless of BMP12 treatment. Thus, CTGF may promote the expression of tenocyte lineage markers while simultaneously repressing differentiation of other cellular phenotypes. This bodes well for the use of CTGF in therapeutic applications to encourage repair of injured tendons without disordering tenocyte differentiation pathways.

BMP molecules signal via type I and type II serine/threonine kinase receptors which form multimeric complexes to phosphorylate and activate Smad proteins for translocation to the nucleus where they exert transcriptional effects. Type II receptors bind BMP ligands, but require association with one or more type I receptors for signal transduction to occur [20]. Two classes of type I receptors have been characterized, BMPR-Ia and BMPR-Ib, with several different Alk receptor isoforms representative of each class. To determine which type I receptor is involved in BMP12 signal transduction, we utilized LDN-1933198, an inhibitor of BMPR-Ia receptors Alk2, Alk3 and Alk6, and SB-505124, which inhibits the BMPR-Ib receptors Alk4, Alk5 and Alk7. Rat TSCs grown in the presence of each inhibitor were stimulated with BMP12 and examined for phosphorylation of Smad1/5/8 proteins. We observed dose-dependent inhibition of BMP12-induced Smad phosphorylation with LDN-193198 but not with SB-505124, suggesting that BMPR-Ia receptors transduce BMP12 signals in rat TSCs. Again, CTGF expression appears to be required to fully activate Smad signaling, since Smad phosphorylation, as well as total Smad protein, were inhibited when CTGF expression was knocked down using the CTGF shRNA vector.

Surprisingly, BMP12 treatment stimulated a large increase in BMPR-Ib mRNA expression compared to untreated control cells, which was also suppressed following CTGF knockdown. In contrast, increased expression of BMPR-Ia was not observed following BMP12 stimulation, indicating that BMP12-induced Smad1/5/8 phosphorylation was not mediated by changes 
in receptor expression. The significance of BMPR-Ib in tenocyte differentiation, given that it does not appear to mediate Smad activation, will need to be explored in future studies.

The ability of CTGF to enhance or maintain activity of BMP12 may depend on a physical interaction between these two proteins. CTGF has been reported to bind directly BMP2 [35] and also to BMP4 and TGF- $\beta$, where it has inhibitory and enhancing effects on receptor binding, respectively [36]. The interaction occurs at the cysteine-rich CR domain of CTGF, a module found in other BMP regulatory proteins like chordin, which antagonizes BMP receptor binding in fruit flies [37]. We used chemical crosslinking experiments to show a direct interaction between BMP12 and full-length CTGF or its CR domain, but not with the CT domain. The mechanism by which CTGF binding modulates BMP12 activity is unknown, but we intend in future experiments to test the possibility that the interaction enhances either receptor binding or receptor kinase activity.

In conclusion, BMP12 signaling plays an important role in the differentiation and development of tenocytes, in which signaling is transduced by BMPR-Ia receptors and activates the Smad1/5/8 pathway. CTGF may promote the stimulatory effect of BMP12 on tenocyte lineage differentiation markers through physical interaction at the CR domain. CTGF thus has therapeutic potential for the treatment of tendon injury and tendinopathy.

\section{Acknowledgments}

This work was supported by the Young Scientists Fund of the National Nature Science Foundation of China (grant nos. 81401831) and the National Nature Science Foundation of China (grant nos. 81230040).

\section{Disclosure Statement}

There is no competing financial interests exist among any authors in relation to the submission.

\section{References}

1 Liu JP, Chen L, Tao X, Tang K: Phosphoinositide 3-kinase/akt signaling is essential for prostaglandin e2induced osteogenic differentiation of rat tendon stem cells. Biochem Biophys Res Commun 2013;435:514519.

2 Hast MW, Zuskov A, Soslowsky LJ: The role of animal models in tendon research. Bone Joint Res 2014;3:193-202.

3 Shen H, Gelberman RH, Silva MJ, Sakiyama-Elbert SE, Thomopoulos S: Bmp12 induces tenogenic differentiation of adipose-derived stromal cells. PLoS One 2013;8:e77613.

4 Lee CH, Shah B, Moioli EK, Mao JJ: Ctgf directs fibroblast differentiation from human mesenchymal stem/ stromal cells and defines connective tissue healing in a rodent injury model. J Clin Invest 2010;120:33403349.

5 Bi Y, Ehirchiou D, Kilts TM, Inkson CA, Embree MC, Sonoyama W, Li L, Leet AI, Seo BM, Zhang L, Shi S, Young MF: Identification of tendon stem/progenitor cells and the role of the extracellular matrix in their niche. Nat Med 2007;13:1219-1227.

6 Rui YF, Lui PP, Li G, Fu SC, Lee YW, Chan KM: Isolation and characterization of multipotent rat tendonderived stem cells. Tissue Eng Part A 2010;16:1549-1558.

7 Zhang J, Wang JH: Production of pge(2) increases in tendons subjected to repetitive mechanical loading and induces differentiation of tendon stem cells into non-tenocytes. J Orthop Res 2010;28:198-203.

-8 Zhang J, Wang JH: Characterization of differential properties of rabbit tendon stem cells and tenocytes. BMC Musculoskelet Disord 2010;11:10. 


\section{Cellular Physiology Cell Physiol Biochem 2015;35:1831-1845

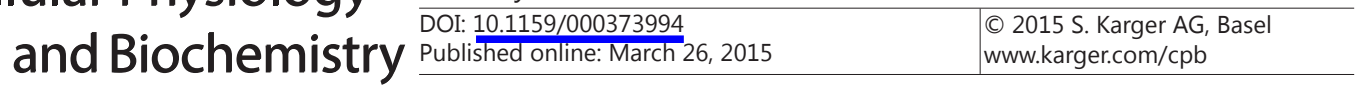

Liu et al.: Factors Regulating Tenogenic Differentiation

-9 Schweitzer R, Chyung JH, Murtaugh LC, Brent AE, Rosen V, Olson EN, Lassar A, Tabin CJ: Analysis of the tendon cell fate using scleraxis, a specific marker for tendons and ligaments. Development 2001;128:38553866.

10 Maeda T, Sakabe T, Sunaga A, Sakai K, Rivera AL, Keene DR, Sasaki T, Stavnezer E, Iannotti J, Schweitzer R, Ilic D, Baskaran H, Sakai T: Conversion of mechanical force into tgf-beta-mediated biochemical signals. Curr Biol 2011;21:933-941.

11 Pryce BA, Watson SS, Murchison ND, Staverosky JA, Dunker N, Schweitzer R: Recruitment and maintenance of tendon progenitors by tgfbeta signaling are essential for tendon formation. Development 2009;136:1351-1361.

12 Schiele NR, Marturano JE, Kuo CK: Mechanical factors in embryonic tendon development: Potential cues for stem cell tenogenesis. Curr Opin Biotechnol 2013;24:834-840.

13 Edom-Vovard F, Schuler B, Bonnin MA, Teillet MA, Duprez D: Fgf4 positively regulates scleraxis and tenascin expression in chick limb tendons. Dev Biol 2002;247:351-366.

14 Shukunami C, Oshima Y, Hiraki Y: Chondromodulin-i and tenomodulin: A new class of tissue-specific angiogenesis inhibitors found in hypovascular connective tissues. Biochem Biophys Res Commun 2005;333:299-307.

15 Tan Q Lui PP, Rui YF, Wong YM: Comparison of potentials of stem cells isolated from tendon and bone marrow for musculoskeletal tissue engineering. Tissue Eng Part A 2012;18:840-851.

16 Storm EE, Huynh TV, Copeland NG, Jenkins NA, Kingsley DM, Lee SJ: Limb alterations in brachypodism mice due to mutations in a new member of the tgf beta-superfamily. Nature 1994;368:639-643.

17 Wolfman NM, Hattersley G, Cox K, Celeste AJ, Nelson R, Yamaji N, Dube JL, DiBlasio-Smith E, Nove J, Song JJ, Wozney JM, Rosen V: Ectopic induction of tendon and ligament in rats by growth and differentiation factors 5, 6, and 7, members of the tgf-beta gene family. J Clin Invest 1997;100:321-330.

-18 Forslund C, Rueger D, Aspenberg P: A comparative dose-response study of cartilage-derived morphogenetic protein (cdmp)-1, -2 and -3 for tendon healing in rats. J Orthop Res 2003;21:617-621.

19 Lou J, Tu Y, Burns M, Silva MJ, Manske P: Bmp-12 gene transfer augmentation of lacerated tendon repair. J Orthop Res 2001;19:1199-1202.

-20 Herpin A, Cunningham C: Cross-talk between the bone morphogenetic protein pathway and other major signaling pathways results in tightly regulated cell-specific outcomes. FEBS J 2007;274:2977-2985.

21 Lee KS, Hong SH, Bae SC: Both the smad and p38 mapk pathways play a crucial role in runx2 expression following induction by transforming growth factor-beta and bone morphogenetic protein. Oncogene 2002;21:7156-7163.

22 Lin GL, Hankenson KD: Integration of bmp, wnt, and notch signaling pathways in osteoblast differentiation. J Cell Biochem 2011;112:3491-3501.

23 Nishimura R, Hata K, Matsubara T, Wakabayashi M, Yoneda T: Regulation of bone and cartilage development by network between bmp signalling and transcription factors. J Biochem 2012;151:247-254. Ali AT, Hochfeld WE, Myburgh R, Pepper MS: Adipocyte and adipogenesis. Eur J Cell Biol 2013;92:229-236. de Mos M, Koevoet WJ, Jahr H, Verstegen MM, Heijboer MP, Kops N, van Leeuwen JP, Weinans H, Verhaar JA, van Osch GJ: Intrinsic differentiation potential of adolescent human tendon tissue: An in-vitro cell differentiation study. BMC Musculoskelet Disord 2007;8:16.

-26 Edom-Vovard F, Duprez D: Signals regulating tendon formation during chick embryonic development. Dev Dyn 2004;229:449-457.

-27 Brent AE, Tabin CJ: Developmental regulation of somite derivatives: Muscle, cartilage and tendon. Curr Opin Genet Dev 2002;12:548-557.

28 Tickle C: Molecular basis of vertebrate limb patterning. Am J Med Genet 2002;112:250-255.

29 Bradham DM, Igarashi A, Potter RL, Grotendorst GR: Connective tissue growth factor: A cysteine-rich mitogen secreted by human vascular endothelial cells is related to the src-induced immediate early gene product cef-10. J Cell Biol 1991;114:1285-1294.

30 Brunner A, Chinn J, Neubauer M, Purchio AF: Identification of a gene family regulated by transforming growth factor-beta. DNA Cell Biol 1991;10:293-300.

31 Ryseck RP, Macdonald-Bravo H, Mattei MG, Bravo R: Structure, mapping, and expression of fisp-12, a growth factor-inducible gene encoding a secreted cysteine-rich protein. Cell Growth Differ 1991;2:225233. 


\section{Cellular Physiology and Biochemistry}

Liu et al.: Factors Regulating Tenogenic Differentiation

32 Mundy C, Gannon M, Popoff SN: Connective tissue growth factor (ctgf/ccn2) negatively regulates bmp-2 induced osteoblast differentiation and signaling. J Cell Physiol 2014;229:672-681.

-33 Kireeva ML, Mo FE, Yang GP, Lau LF: Cyr61, a product of a growth factor-inducible immediate-early gene, promotes cell proliferation, migration, and adhesion. Mol Cell Biol 1996;16:1326-1334.

34 Moussad EE, Brigstock DR: Connective tissue growth factor: What's in a name? Mol Genet Metab 2000;71:276-292.

-35 Maeda A, Nishida T, Aoyama E, Kubota S, Lyons KM, Kuboki T, Takigawa M: Ccn family 2/connective tissue growth factor modulates bmp signalling as a signal conductor, which action regulates the proliferation and differentiation of chondrocytes. J Biochem 2009;145:207-216.

36 Abreu JG, Ketpura NI, Reversade B, De Robertis EM: Connective-tissue growth factor (ctgf) modulates cell signalling by bmp and tgf-beta. Nat Cell Biol 2002;4:599-604.

37 Garcia Abreu J, Coffinier C, Larrain J, Oelgeschlager M, De Robertis EM: Chordin-like cr domains and the regulation of evolutionarily conserved extracellular signaling systems. Gene 2002;287:39-47.

38 Chen CH, Cao Y, Wu YF, Bais AJ, Gao JS, Tang JB: Tendon healing in vivo: Gene expression and production of multiple growth factors in early tendon healing period. J Hand Surg Am 2008;33:1834-1842.

39 Ni M, Lui PP, Rui YF, Lee YW, Lee YW, Tan Q, Wong YM, Kong SK, Lau PM, Li G, Chan KM: Tendon-derived stem cells (tdscs) promote tendon repair in a rat patellar tendon window defect model. J Orthop Res 2012;30:613-619.

40 Ruzzini L, Longo UG, Rizzello G, Denaro V: Stem cells and tendinopathy: State of the art from the basic science to clinic application. Muscles Ligaments Tendons J 2012;2:235-238.

-41 Safadi FF, Xu J, Smock SL, Kanaan RA, Selim AH, Odgren PR, Marks SC Jr, Owen TA, Popoff SN: Expression of connective tissue growth factor in bone: Its role in osteoblast proliferation and differentiation in vitro and bone formation in vivo. J Cell Physiol 2003;196:51-62. 